\title{
EL LATINOAMERICANISMO COMO PENSAMIENTO DESCOLONIZADOR
}

\begin{abstract}
Alberto Saladino García $\left(^{*}\right)^{1}$
RESUMEN

En este artículo se explica la génesis de la filosofía del latinoamericanismo, entendida ésta como praxis descolonizadora. Para el efecto se valora el impacto de la lucha de hombres y mujeres en la fundamentación de la identidad del ser del latinoamericano y de América Latina desde una posición libertaria. Para sustentar esta perspectiva libertadora primero se presentan los argumentos justificadores del colonialismo, luego, la crítica al colonialismo, el reconocimiento de América como matria y el respaldo al ejercicio de la soberanía, todo ello amparado en los datos de la historia latinoamericana y en el inventario de nuestros aportes a la cultura mundial.

Palabras clave:

Colonialismo - cultura - filosofía - latinoamericanismo - soberanía.

(*) Doctor en Estudios Latinoamericanos de la Universidad Nacional Autónoma de México. Profesor-investigador de la Facultad de Humanidades de la Universidad Autónoma del Estado de México.

Artículo recibido el 20 de marzo de 2010. Aceptado por el Comité Editorial el 29 de septiembre de 2010.

Correo electrónico: asaladinog@uaemex.mx.

${ }^{1}$ Autor de nueve libros y compilador de ocho libros.
\end{abstract}




\begin{abstract}
In this article is explained the genesis of the philosophy of the latinoamericanismo as descolonizing praxis. For this purpose is studied the impact of the struggle of men and women on the foundations of the identity of being Latin American and Latin America since a libertarian position. To support this liberator perspective, appear first the arguments justifying colonialism, afterwords, the critique of colonialism, the recognition of America as matria and the support for the exercise of sovereignty, all based on facts of the Latin American history and the inventory of us contributions to world culture.
\end{abstract}

\title{
Keywords:
}

Colonialism - culture - philosophy - latinoamericanismo - overeignty.

Sistematizar la génesis de la filosofía del latinoamericanismo constituye una tarea imprescindible para recuperar los saldos de la lucha de hombres y mujeres preclaros, interesados en fundamentar la identidad y ser del latinoamericano y de América Latina. Tal tarea se erigió, históricamente, en un verdadero reto a asumir como respuesta natural al colonialismo intelectual generado por la conquista ibérica el cual se codificó mediante la descalificación de las producciones de las culturas precolombinas, primero, y de los criollos y mestizos, más tarde, así como de la naturaleza americana.

\section{JUSTIFICACIÓN COLONIALISTA}

Son diversas las manifestaciones del colonialismo económico, social, político y cultural, padecido por los habitantes del continente americano desde el siglo XVI, y cuyas expresiones las han evidenciado estudiosos latinoamericanos, las cuales se pueden identificar, por ejemplo para el caso que nos interesa, con la negación de la creatividad intelectual, justificada abundantemente, por científicos extranjeros, europeos para señas precisas.

Esas descalificaciones de los aportes de los americanos a la cultura han tenido a representantes de distinta calidad intelectual a lo largo de los últimos cinco siglos. Puede iniciarse el inventario con los mismos conquistadores que destruyeron los códices, quipus y demás creaciones de las culturas preamericanas; las calumnias del virrey del Perú, Toledo y Sarmiento de Gamboa sobre la cultura inca o los religiosos que negaron las cualidades intelectuales de personajes como Juana Inés de Asbaje y Ramírez en el siglo XVII; la negación de la madurez de la naturaleza, las sociedades y el hombre del Nuevo Mundo al sustentar tesis acerca de la inferioridad americana como lo patentiza el caso de George Louis Leclerc, conde de Buffon, cuyas interpretaciones fueron ampliamente propaladas por Cornélius de Pauw y reiteradas por David Hume, Joseph-Francois de Montesquieu, Francois-Marie Arout de Voltaire, 
Guillaume-Thomas-Francois Raynal y William Robertson, durante el siglo XVIII; Federico Hegel acerca de la identificación de América como tierra nueva y por ende lugar sin historia, porque sus habitantes originarios los vinculó como expresiones de la naturaleza misma y porque apreció su situación sociocultural como mero eco del viejo mundo y, en consecuencia, expresión de una vitalidad ajena ${ }^{3}$, o por la descalificación hecha por Federico Engels al comentar el despojo de Estados Unidos a México, de más de la mitad de su territorio, en los términos siguientes:

En América hemos presenciado la conquista de México, la que nos ha complacido. Constituye un progreso, también, que un país ocupado hasta el presente exclusivamente de sí mismo, desgarrado por perpetuas guerras civiles e impedido de todo desarrollo, un país que en el mejor de los casos estaba a punto de caer en el vasallaje industrial de Inglaterra, que un país semejante sea lanzado por la violencia al movimiento histórico. Es en interés de su propio desarrollo que México estará en el futuro bajo la tutela de los Estados Unidos. Es en interés del desarrollo de toda América que los Estados Unidos, mediante la ocupación de California, obtienen el predominio sobre el Océano Pacífico... ${ }^{4}$

Tales interpretaciones sobre América Latina fueron desplegadas a lo largo del siglo XIX; apreciaciones que no cambiaron en pleno siglo XX como lo prueban posiciones incluso de especialistas de la cultura latinoamericana, tal es el caso del estadounidense Irving A. Leonard quien objeta la asociación de las expresiones Hispanoamérica y ciencia al sustentar:

Al meditar que vivimos en un mundo de ciencia y reflexionar sobre mi largo interés en la América Española, me impresiona la casi completa ausencia de asociación de dos abstracciones: la ciencia y la América Española. Contemplando el maravilloso desfile que nos ofrece el desarrollo de la ciencia y de la tecnología durante el pasado medio milenio, las grandes realizaciones parecen ser creación principal, aunque no exclusivamente, de los pueblos de Europa y, más recientemente, de la América del Norte. Los nombres históricos de hombres de ciencia que al punto acuden a nuestra memoria son ingleses, italianos, alemanes, franceses, escandinavos y norteamericanos; rara vez son hispánicos, y aún menos hispanoamericanos. Por mucho que lo intentemos, nos resultará difícil mencionar a un hombre de ciencia grande e imaginativo asociado a las tierras de las culturas hispánicas, que pudiera compararse a Leonardo da Vinci o a Copérnico, o inventores como Thomas Alva Edison y Alexander Graham Bell. ¿Por qué?... ${ }^{5}$

La respuesta a su pregunta la buscó en la cultura de tradición escolástica implantada y en las condiciones geográficas, pero al final de cuentas su planteamiento exhibe la persistencia del coloniaje intelectual propalado sobre los latinoamericanos, como queda demostrado y ha sido la constante a lo largo de medio milenio de occidentalización de América Latina.

\footnotetext{
2 Ver Antonello Gerbi, La disputa del Nuevo Mundo. Historia de una polémica 1750-1900, $1^{a}$ reimpresión, México, Fondo de Cultura Económica, 1993, pp. 7-409 y Michéle Duchet, Antropología e historia en el siglo de Las Luces, 2a edición, México, Siglo XXI Editores, 1984, pp. 243-325.

${ }_{3}^{3}$ Cfr. Federico Hegel, Filosofía de la historia, Barcelona, Ediciones Zeus, 1971, pp. 94 - 105 - 110.

4 Kart Mar y Friedrich Engels, Materiales para la historia de América Latina, México, Cuadernos de Pasado y Presente 30, 1975 , p. 183.

${ }_{5}$ Irving A. Leonard, Ensayos y semblanzas: bosquejos históricos y literarios de la América colonial, México, Fondo de Cultura Económica, 1990, p. 11.
} 


\section{PENSAMIENTO DESCOLONIZADOR}

La respuesta al colonialismo intelectual vino manifestándose, igualmente, como una constante de los estudiosos en estas tierras, al concientizar dicha situación desde el momento mismo de su génesis. Inició con la labor de religiosos como Bartolomé de Las Casas, quien sentó las bases para el reconocimiento de los habitantes de la época prehispánica y sus descendientes como humanos, en igualdad de posibilidades y potencialidades, tanto físicas e intelectuales, con los habitantes del viejo mundo, así como la actividad gnoseológica de las producciones de Mesoamérica desplegada por Bernardino de Sahagún, y de la zona andina por Joseph de Acosta, en el siglo XVI; el quehacer intelectual tan notorio del Inca Garcilaso de la Vega a partir de la publicación de La Florida del Inca (1605) -al mismo tiempo en que Miguel de Cervantes publicaba Don Quijote de la Mancha- y los Comentarios reales (1609), la obra magistral de Juana Inés de Asbaje y Ramírez con su Primero sueño, Carta atenagórica y Respuesta a Sor Filotea de la Cruz, o la obra de los científicos Carlos de Sigüenza y Góngora y Pedro de Peralta Barnuevo y Rocha y Benavides, durante el siglo XVII y en el caso de éste prolongada en la primera mitad del siglo XVIII, así como la obra de laicos y religiosos criollos durante la segunda mitad a través de los casos de Francisco Javier Alegre, Antonio de Alcedo, José Antonio Alzate y Ramírez, Francisco Arango y Parreño, José Ignacio Bartolache, Cosme Bueno, José Agustín Caballero, Francisco José de Caldas y Tenorio, Francisco Javier Clavijero, José Joaquim da Cunha de Azeredo Coutinho, Isidoro de Celis, Juan Benito Díaz de Gamarra, Juan José de Eguiara y Eguren, José Landívar, Antonio León y Gama, José Eusebio Llano y Zapata, José Mariano Mociño, Juan Ignacio Molina, Francisco Antonio Moreno y Escandón, Simón Rodríguez, Tomás Romay, Buenaventura Suárez, Hipólito Unanue, Joaquín Velásquez de León, José Mariano da Conceicâo Veloso, etc.

Ellos sentaron los fundamentos mediante los cuales se consolidó el proceso de autoconocimiento, que se venía desarrollando desde el siglo XVI, al poco rato de la institucionalización de la cultura y la educación occidentales en esta parte del mundo, lo cual engendró cuestiones y preocupaciones que dieron origen a la génesis de la filosofía del latinoamericanismo como consecuencia del triunfo de las luchas de independencia a lo largo del siglo XIX, destacadamente por la obra intelectual de los mismos próceres como Francisco de Miranda, Simón Bolívar, José María Morelos y Pavón, Francisco Bilbao, Antonio Nariño, y de sus continuadores en los años posteriores como Juan Bautista Alberdi, Benito Juárez, José Martí, José Ingenieros, José Victorino Lastarria, Benjamín Vicuña Mackenna y José Enrique Rodó, etc.

Entonces la centuria decimonónica sentó las bases para la emergencia de la filosofía del latinoamericanismo, esto es, del pensamiento descolonizador, por crítico, cuestionador y alternativo. Tal proceso sobre la génesis de la construcción de la filosofía del latinoamericanismo se consolidó con la obra de intelectuales de todo el continente, a lo largo del siglo XX, destacadamente gracias a la labor de intelectuales como Arturo Ardao, Guillermo Francovich, Paulo Freire, Risieri Frondizi, José Gaos, Pedro Henríquez Ureña, Manfredo Kempff Mercado, Alejandro Korn, Rodolfo Kush, Ernesto Mayz Ballenilla, Domingo Miliani, Mariano Picón Salas, Francisco Romero, 
Augusto Salazar Bondy, Ricaurte Soler, José Vasconcelos, Abelardo Villegas, Gregorio Weinberg, Leopoldo Zea, etc.

\section{RASGOS DEL PENSAMIENTO DESCOLONIZADOR}

De modo que a la par del proceso de occidentalización de América Latina emergió la preocupación por impulsar otra lectura de nuestra historia y con base en ella se ha venido sistematizando el latinoamericanismo como pensamiento descolonizador y con base en él se ha intensificado la lucha contra el colonialismo, en sus más diversas manifestaciones.

En consecuencia la codificación del conocimiento de América desde una posición latinoamericanista, esto es libertaria, requiere atenderla más exhaustivamente, con el propósito de enriquecer las fuentes y tópicos de la filosofía de nuestra América. Para el efecto desgloso algunos de sus rasgos.

1. Crítica al colonialismo. El latinoamericanismo como pensamiento descolonizador emergió antes de que el término apareciera. De manera evidente lo testimonian las actitudes intelectuales y aportes de criollos, laicos y religiosos, de la época colonial, en particular durante la segunda mitad del siglo XVIII. Lo desarrollaron en Europa los expulsos jesuitas y fue militante esa actitud en la amplia nómina de ilustrados latinoamericanos, en particular cito los casos paradigmáticos de los religiosos Juan José de Eguiara y Eguren y José Antonio Alzate y Ramírez.

En efecto, José de Eguiara y Eguren, en su interés por evidenciar la labor intelectual de los americanos, se echó a cuestas la obra titánica de elaborar la primera Bibliothecae mexicanae o Historia de los varones eruditos que en la América Boreal nacidos o que, en otra tierra procreados, por virtud de mansión o estudios en ésta arraigados, en cualquier lengua algo por escrito legaron... y con cualquier género de escritos publicados o inéditos, egregiamente florecieron, cuyo tomo primero apareció en latín en 1755, donde puso de manifiesto la preocupación por ensalzar la creatividad cultural de una parte de América ${ }^{6}$. Su obra sustanció una de las reacciones más nítidas contra las expresiones de colonialismo intelectual del viejo mundo.

Otro tipo de reacción que enfrentó al colonialismo intelectual la promovió José Antonio Alzate al señalar los errores de muchas informaciones de científicos europeos y rechazó la imposición de las nomenclaturas botánica de Carlos Lineo y química de Antonio Lorenzo Lavoisier en la enseñanza en Nueva España, como mero efecto del dominio occidental, incluso en el primer caso, su cuestionamiento lo soportó con la proposición de los contenidos y criterios de la taxonomía nahuatl, de origen

\footnotetext{
${ }^{6}$ José de Eguiara y Eguren, Biblioteca mexicana o Historia de los varones eruditos que en la América Boreal nacidos o que, en otra tierra procreados, por virtud de mansión o estudios en ésta arraigados, en cualquier lengua algo por escrito legaron, principalmente de aquellos que en dilatar y favorecer la fe católica y la piedad con sus hazañas y con cualquier género de escritos publicados o inéditos, egregiamente florecieron, México, Universidad Nacional Autónoma de México, Prólogo y versión en español de Benjamín Fernández Valenzuela. Estudio preliminar, notas, apéndices, índices y coordinación general de Ernesto de la Torre Villar con la colaboración de Ramiro Navarro de Anda, 1986, Tomo Primero, 543 pp.
} 
prehispánico, para hacer patente la creatividad de los americanos ${ }^{7}$.

2. América como matria. Uno de los mayores esfuerzos hechos para diferenciar a las sociedades americanas de las europeas lo constituyó la labor de los próceres de la independencia, con Simón Bolívar a la cabeza, al pugnar por la liberación y unión del espacio que hoy denominamos América Latina. Con conocimientos eruditos de nuestra historia y geografía, que le sirvieron de respaldo a su lucha anticolonial, tuvo plena conciencia de su importancia para despertar el amor a la tierra americana ${ }^{8}$, por lo cual los fomentó.

Una postura idéntica la descollaron científicos comprometidos con la independencia como sería el caso del neogranadino Francisco José de Caldas quien estimuló el conocimiento científico con propósitos de compromiso para el bienestar social al expresar:

Observar el cielo por observarlo sería una ocupación honesta, pero no pasaría de una curiosidad estéril que llenase los momentos del hombre ocioso y acomodado. Este observador sería inútil, y la Patria lo miraría como un consumidor de quien no esperaba nada. Nosotros no queremos representar este papel en la sociedad: queremos que nuestros trabajos astronómicos mejoren nuestra geografía, nuestros caminos y nuestro comercio... ${ }^{9}$

De lo cual se deriva que existiera un interés creciente por exaltar lo americano, a partir del conocimiento de su territorio y por eso resultó natural que se identificara entonces a América como la verdadera matria, según la había bautizado el Mercurio Peruano unos años antes.

3. América espacio soberano. Igualmente los próceres de la independencia y los diseñadores de nuestras naciones enfatizaron las diferencias con las metrópolis mediante el empleo de diversas expresiones para denominar a esta región, como América Septentrional, América del Sur, etc., para testimoniar el ejercicio de su soberanía, de construir sociedades libres, pueblos independientes.

Así José María Morelos y Pavón dispuso en los Sentimientos de la Nación, artículo “5 Que la soberanía dimana inmediatamente del pueblo, el que sólo quiere depositarla en el Supremo Congreso Nacional Americano, compuesto de representantes de las provincias en igualdad de números"10.

\footnotetext{
7 Cfr. José Antonio Alzate y Ramírez, Gacetas de literatura de México, Puebla, Imprenta del Hospital de San Pedro y San Pablo, 1831, 4 tomos y, de manera específica, Patricia Aceves Pastrana, “La difusión de la ciencia en la Nueva España en el siglo XVIII: la polémica en torno a la nomenclatura de Linneo y Lavoisier", Quipu, Vol. 4, N 3, 1987, pp. 357-385.

${ }^{8}$ Simón Bolívar, Carta de Jamaica, CCYDEL/Facultad de Filosofía y Letras de la UNAM, Latinoamérica, Cuadernos de cultura latinoamericana 1, 1978, $32 \mathrm{pp}$.

9 Francisco José de Caldas, "Preliminares para el almanaque de 1811", Obras completas, Bogotá Universidad Nacional de Colombia, 1966, p. 402.

${ }^{10}$ José María Morelos y Pavón, "Sentimientos a la Nación", Martha Celada Castillo y Ruvalcaba, Antología de lecturas de Historia de México, Universidad Nacional Autónoma de México, 1995, p. 96.
} 
Benito Juárez, con su indeclinable espíritu de lucha, en su obra puso de manifiesto su mística contra cualquier tipo de colonialismo y con base en su denodada y triunfal acción a favor de la soberanía aportó al mundo el apotegma que lo inmortalizó: “Que el pueblo y el gobierno respeten los derechos de todos. Entre los individuos, como entre las naciones, el respeto al derecho ajeno es la paz"11.

4. Nuestra América como expresión de su historia. La obra de intelectuales de la talla de José Martí, Juan Bautista Alberdi, José Enrique Rodó, etc., pone de relieve que la historia de nuestra América, la América Latina, tiene un origen común, la cual aporta las bases de la identidad de los pueblos de la región, por lo que convocan a profundizar su conocimiento para el efecto.

De manera que estos intelectuales no sólo plantearon la necesidad de resaltar todas las manifestaciones de la cultura humana, sino de forjar la identidad cultural de Latinoamérica adaptando algunos aspectos a las circunstancias históricas y, sobre todo, al priorizar el conocimiento riguroso de nuestras sociedades. La obra de José Martí es ejemplar al respecto y las diferentes expresiones utilizadas para referirse a ella delatan su convicción descolonizadora y antiimperialista. Ciertamente el empleo reiterativo de las expresiones "América", "Madre América", "Nuestra América", "Nuestra América fabulosa" "nuestra América desinteresada", como la llamó indistintamente, y al definirse: “De América soy hijo; a ella me debo. Y de América, cuya revelación, sacudimiento y fundación urgente me consagro"12, posibilitan considerarlo, como quien más, forjador del latinoamericanismo como pensamiento libertario, al entregar su vida a la lucha para poner fin al colonialismo, coadyuvar a la independencia mental y propugnar el ideal bolivariano de unidad latinoamericana.

5. Espacio de aportes culturales. América Latina, en el siglo XX, emergió como una región del mundo desde la cual se contribuye al desarrollo cultural de la humanidad. Este reconocimiento se lo ganó por la generación más brillante de artistas, científicos, humanistas y tecnólogos comprometidos con la liberación intelectual, y que en su mayor parte vivieron durante la segunda mitad de dicha centuria.

En campos diversos de las artes dieron origen a nuevas corrientes, variedad de estilos y enfoques. Por ejemplo, en el ámbito de la literatura forjaron el género de lo real maravilloso; en el cine la época de oro; en la pintura el muralismo de compromiso social; en la arquitectura la recuperación de estilos vernáculos; en el teatro se trascendió los muros de las aulas y foros cerrados mediante el cultivo del arte como compromiso social, etc.

En el ámbito de la ciencia aparecieron los grandes esfuerzos por usar el conocimiento racional para amortiguar los problemas socioeconómicos. De esta forma se dieron origen a las políticas científicas que posibilitaron premios nobel, como el del argentino Bernardo Houssay y el mexicano Mario Molina, o avances significativos en la

\footnotetext{
11 Benito Juárez, Antología, México, Universidad Nacional Autónoma de México, Biblioteca del Estudiante Universitario $\mathrm{N}^{\circ} 99,1993$, p. 227.

12 José Martí, Política de nuestra América, México, Siglo XXI, 1999, p. 58.
} 
normalización de la investigación científica y las notorias innovaciones tecnológicas que tiene a Brasil y Cuba como ejemplos.

En el campo de las humanidades mediante el surgimiento de la teología de la liberación, la teoría de la dependencia, la sociología de la explotación, la pedagogía de la liberación y la filosofía de la liberación. Posturas teóricas dedicadas a descolonizar las explicaciones acerca de la realidad latinoamericana. Dentro de esa nómina destacó Leopoldo Zea, por sus acciones de promotor de instituciones y sus reflexiones fecundas, como organizador de la teoría y praxis del latinoamericanismo, de la filosofía del latinoamericanismo.

Entonces, Leopoldo Zea concretó la filosofía del latinoamericanismo mediante la interpretación rigurosa de la realidad de nuestra América y con base en ella propugnó la integración en la libertad como medio para hacer frente a la problemática social irresuelta. Dicho de otra manera, en su amplia obra teórica y por su denodada labor de institucionalización del estudio de la realidad de las sociedades latinoamericanas, lo llevó a construir América Latina como objeto de estudio. Esto es, demarcó epistemológicamente una nueva área gnoseológica como mecanismo para trascender el colonialismo en sus diversas manifestaciones. Así nos legó como herencia el latinoamericanismo, como filosofía sin más en estricto sentido, esto es como pensamiento descolonizador, por crítico, libertario y alternativo.

En consecuencia, quienes hoy, desde la academia, acompañaron las luchas bolivarianas de los tiempos que corren, por su adscripción al latinoamericanismo, como Adriana Arpini, Hugo Biagini, Carmen Bohórquez, Horacio Cerutti, Carlos Cullen Soriano, Eduardo Devés-Valdés, Enrique Dussel, Estela Fernández Nadal, Raúl Fornet-Betancourt, Pablo Guadarrama González, Clara Alicia Jalif de Bertranou, Francisco Miró Quesada, Javier Ocampo López, Dina V. Picotti C., María Luisa Rivara de Tuesta, Arturo Andrés Roig, Alberto Saladino García, Juan Carlos Scannone, Alejandro Serrano Caldera, etc. tenemos la responsabilidad de amplificar el programa de la filosofía del latinoamericanismo para vislumbrar nuevos horizontes y derroteros de los pueblos de América Latina con la perspectiva de consumar los ideales de los hombres y mujeres que iniciaron el proceso de independencia, entendido como praxis de la soberanía popular, hace doscientos años, y como mínimo homenaje. 